\title{
A new methodology for the development of CSCW systems
}

\author{
P. K. Ray
}

The University of Western Sydney, Nepean

Department of Computing, P.O. Box 10, Kingswood NSW 2747, Australia

Tel: +61296859251, Fax: +61296859245

E-mail:pradeep@st.nepeancs.uws.edu.au

\begin{abstract}
The emerging development of virtual corporations, networks and facilities raises the issue of the management of development processes for future enterprise-wide networked applications. Recently there has been some interesting developments in the area of Computer Supported Cooperative Work (CSCW) that provides a binding between organisational business processes and emerging communication technologies. This paper presents a new methodology for the development of CSCW systems. The commonly used help-desk based cooperative business model has been used for illustration. This paper also presents the application of this methodology as a case study in a large telecommunication organisation. This work lays the foundation for research in the evolution of effective means for the development of future cooperative information systems.
\end{abstract}

\section{Keywords}

CSCW, methodology, help-desk, cooperative systems, enterprise networks, usercentred design 


\section{INTRODUCTION}

The emerging enterprise-wide networks would need to support mission critical business applications, and the cooperation of people across distance, using the latest communication technologies. The management of a large networked enterprise requires the cooperation of people with different expertise within and across organisations. Consequently, cooperative management models, such as help-desk are being adopted in a wide variety of business processes. However, recent studies have shown that the existing automated implementations of these models leave much to be desired (Sachs, 1995). Much of the criticism relates to the lack of understanding of human factors in these processes. Therefore, it is necessary to explore newer techniques for the study and development of cooperative distributed computing applications (Preece, 1994). This paper presents a new approach to the design of applications for networked business enterprises.

This paper starts with a description of Computer Supported Cooperative Work (CSCW) concepts. This leads to our new CSCW system development methodology, called Cooperative systems development Methodology for Enterprise Networks (CoMEN). This is followed by an illustration of this methodology based on its application in a large telecommunication organisation.

\section{CSCW SYSTEM DEVELOPMENT METHODOLOGIES}

" $\mathrm{CSCW}$ is a fusion of the understanding of a business organisation with the possibilities of computer and communication technologies." This definition was developed by Tom Rodden of the Centre for CSCW at Lancaster University (CSCW, 1994). CSCW in its present form took shape in the mid 1980s.

Modern networked enterprises are based on emerging distributed client-server systems. Some of these cooperative systems have distributed groups of development teams, requiring formal groupware and workflow platforms/tools, such as Lotus NOTES (Lotus, 1993).

CSCW in some countries also reflects cultural norms, such as national homogeneity, strong trade unions, and extensive social welfare systems. For example, Scandinavian participatory or collaborative design approach reflects priorities, such as the need to support workers losing automated jobs (Preece, 1994).

The objective of our research is to incorporate these contexts into the development of a methodology for the management of enterprise networks based on CSCW. User-centred CSCW methodologies are now evolving. The major ones are as follows (Preece, 1994).

\section{Soft Systems Methodology}

All human actions take place within wider contexts, or situations. The essential aspect of understanding situations from a systems perspective is to consider the sys- 
tem as a whole (Suchman, 1991), (Winograd,1987). The emphasis of SSM is on understanding the situation in which a perceived problem is thought to lie (Checkland, 1990).

\section{MultiView}

A more prescriptive approach is to embed sociotechnical and soft systems into a more all-encompassing methodology. Multiview is an information systems design methodology which combines these different approaches into a staged, controlled methodology (Avison, 1995). It allows both human as well as technical factors to be considered at the design stage.

\section{Star Life Cycle}

The star stresses rapid prototyping and an incremental development of the final product. Although the titles given to the main stages are different, they represent similar activities to the Waterfall model (Preece, 1994): analysis (Task/functional analysis), requirements specification and design (conceptual/physical design) and implementation. But the process requires considerably more iteration. The requirements, design and the product gradually evolve, become increasingly well defined.

While Soft Systems Methodology focuses on holistic analysis and planning, Multiview is a complete methodology which spans the whole of planning through implementation. The star life cycle focuses primarily on design, and uses the popular iterative processes for the design of CSCW systems.

In addition to SSM, there are a number of other people-oriented methodologies, such as Enid Mumford's Effective Technical and Human Implementation of Computer-based systems (ETHICS) (Avison, 1995). Our methodology, CoMEN uses SSM based approach for the overall systems analysis. It uses Multiview methodology for the design of workflows and the group support software. The following section presents the modelling concepts, and the process of CoMEN.

\section{THE NEW METHODOLOGY: CoMEN}

This methodology consists of two parts:

\section{Scenario Analysis}

This part of CoMEN uses a human centred systems analysis approach, encompassing requirement gathering to an abstract level analysis, based on Checkland's SSM (Checkland, 1990). This part produces an abstract CSCW specification based on a concept called "awareness model". This part of the methodology is described in detail in the subsequent sections of this paper.

\section{System Design}

This part of CoMEN starts with the abstract awareness specification in the previous 


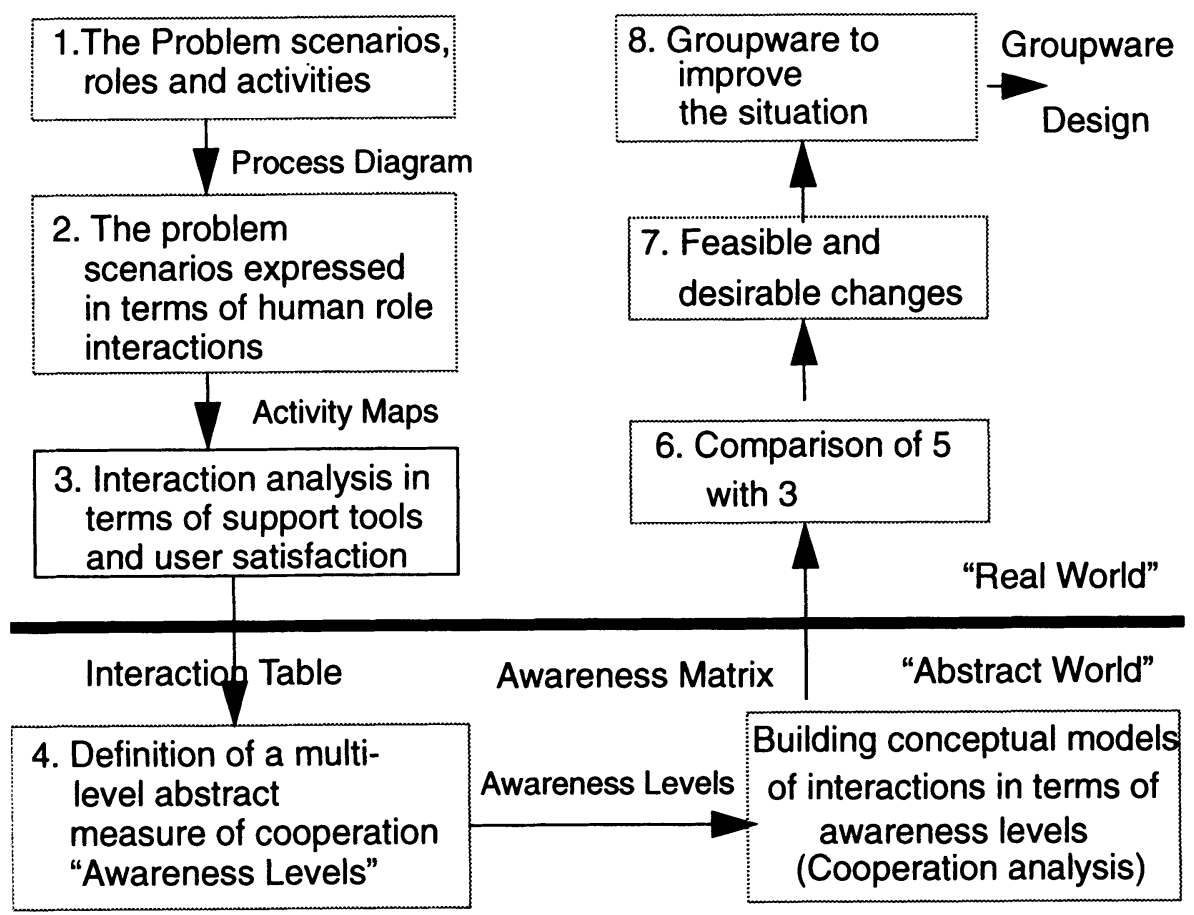

Figure 1 CoMEN Scenario Analysis Process.

stage (scenario analysis), and involves the design, implementation and evaluation of a distributed cooperative system, based on workflows and various distributed object modelling techniques. The approach is similar to Multiview methodology. This part is not discussed in this paper due to space constraints. Details are available at (Ray, 1996).

The Scenario Analysis is a holistic systems analysis process, encompassing the process of requirements collection and analysis of the cooperative management data with a view to designing an abstract cooperative management design specification. In this phase information on human roles, and their interactions is collected from a real organisation. Then process study is undertaken with respect to some representative scenarios (chosen in consultation with people in the organisation under study). For every interaction in a scenario, we draw up a matrix of CSCW support mechanisms (group communication / repositories), and user satisfaction level. Finally, we use an evolving abstract CSCW model based on human "awareness levels" to correlate user satisfaction levels with CSCW support facilities.

The following sections illustrate the scenario analysis part of CoMEN through a case study in a large telecommunication organisation. 


\section{COMEN APPLICATION: A CASE STUDY}

We study cooperative management through a help-desk based troubleticketing application within the context of a distributed computing infrastructure of a telecom service provider.In this case, management personnel cooperate to manage a problem using various network elements. Whenever there is a problem, the customer reports that to the help-desk. The operator then assigns a troubleticket to the problem and assigns it to an appropriate technical person. This person tries to solve the problem and escalates the alarm (calls the EXPERT) if the problem can not be solved within the stipulated time (Johnson, 1992).

Our investigation involved working experience with the major tools (e.g., platforms, simulators, help-desk software) and a series of discussions with people using these tools as part of their jobs in the organisation under study. This helped us with the identification of the human roles and their interaction types (steps 1 and 2 of Figure 1). The results of this phase are summarised in Section 4.1 and Figure 2. The subsequent phases will be involve an analysis based on a new evolving CSCW model, called the awareness model (steps 3-6 of Figure 1).

\subsection{Process Study}

This study was conducted in a real organisation along with people working in a help-desk based network management process, called troubleticketing. This involved the identification of a number of representative scenarios. We then carried out an in-depth study of five scenarios. This paper illustrates the study based on one of these scenarios.

This scenario is now illustrated with a process diagram with corresponding roles and interactions listed in the subsection. In this diagram, each box represents a human role. Communications between roles are represented using directional arrows, the starting at the role initiating the communication. These roles correspond to human roles in a typical network management organisation in a large enterprise.

The Sydney to Perth wide area link, which is on an existing 2 Mbps link on a lower version Network Terminating Unit (NTU), needs to be upgraded to a higher version NTU. For this an outage of 30 minutes is required. This involves the following interactions (Figure 2) amongst roles defined in the previous section:

1. Network management Centre (NMC) Technician discusses with a remote Test Coordinator about a suitable time (Role B/D <---> Role C)

*2. NMC Technician/Expert places change request with proposed time and user impact statement to Change Manager (Role B/D ----> Role G).

*3. Change Manager takes up with the affected Users (Role G -----> Role U).

4. Related Users discuss impact and examine proposed time.

5. User resubmits request to Change Manager with possibly an altered schedule.

6. Change Manager issues Permit To Work to all concerned. 
7. Help-Desk Operator notified before start of work.

8. Help-Desk Operator alerts users regarding start of work.

9. Work coordination between local and remote sites.

10. Check if OK.

11. Help-Desk Operator notified of completion.

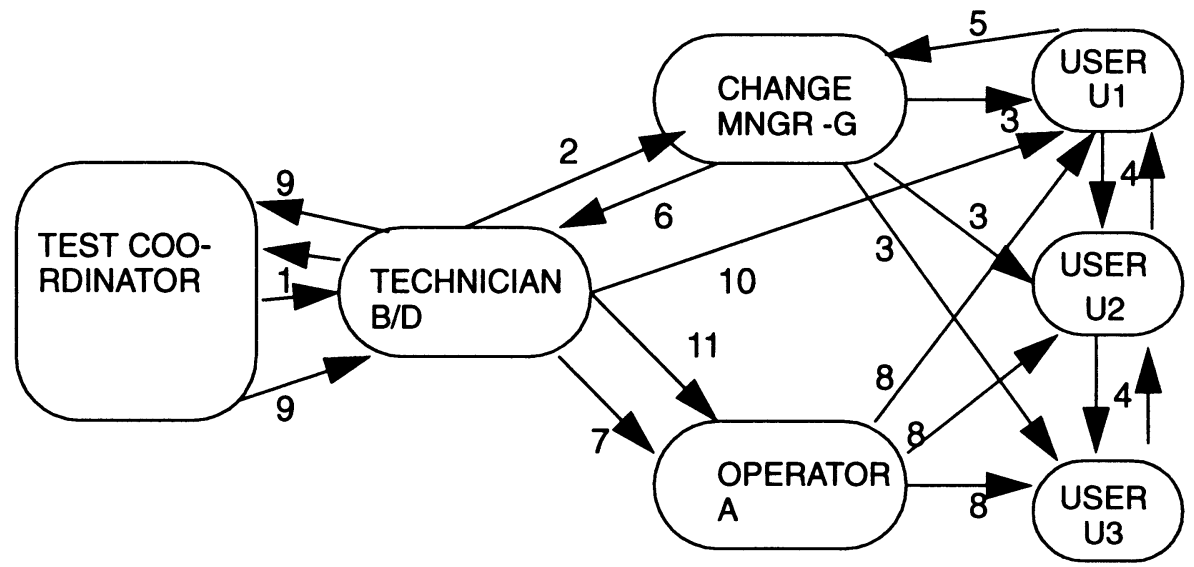

Figure 2 Process Diagram for the Sample Scenario.

The majority of the interactions are not supported by existing automated tools and artifacts. A number of interactions require cooperation of multiple human roles simultaneously.

This scenario is perhaps the simplest of all scenarios studied by us as part of the process study. The interactions listed above are also encountered in other scenarios. Therefore, this paper presents (in the section) the analysis of interactions in this scenario (step 3 of Figure 1).

\section{COLLABORATIVE SERVICE EVALUATION}

Successful collaborative work requires information sharing, knowledge of group and individual activity, and coordination. These factors are important for abstract modelling and design of a CSCW system. (Dourish, 1992) defines a parameter called "awareness" to represent the understanding of the activities of others, which provides a context for one's own activity. Awareness information is required to coordinate group activities in various application domains. While Dourish discusses this concept for a text editing task area, awareness is likely to be useful in a wide variety of cooperative management applications.

We discussed with practising network management professionals the tools and group communication mechanisms in the system under study for all interactions in 
the scenarios studied (Ray, 1996). The objective was to answer the following questions for every interaction:

- What are the group communication systems and tools being used now for the particular interaction?

- What is the level of satisfaction with this type of interaction (This is a value between 0 and 9,0 the lowest satisfaction level, recorded according to the response from people working with the existing system.)?

- If this interaction is not adequately supported now, what type of tools or communication facilities could improve the support?

After collecting this information, we need to perform some abstract modelling as suggested in steps 4 and 5 of Figure 1. This is discussed in the next section.

\section{AWARENESS MODEL}

It is necessary to model all interactions in terms of an abstract measure of cooperation in order to develop a generalised cooperative management framework that would be applicable to a wide variety of businesses.

\section{Abstract CSCW Model for Measuring Cooperation}

"Awareness Levels

*Awareness Transition

*Awareness Matrix for Interactions

*Usability of Awareness Levels

"Awareness vs. Security

\begin{tabular}{l}
\hline Real-Life Scenarios \\
* Help-Desk based Appln. \\
* Interactions \\
* Awareness Required \\
* Awareness Supported \\
* User Satisfaction \\
* Remarks
\end{tabular}

CSCW Design

* Interaction Database

* Group Communication

* Autonomous Agents

*Workflows

* Distributed Objects

Figure 3 Awareness Model.

There is very little reported work on attempts to define measures of awareness. (Benford, 1994) defines two measures "focus" and "nimbus", for interpersonal means of creating awareness. While increased focus makes a person more aware of actions of other members of a group, by increased nimbus a person can make other members of the group more aware of one's actions. We have defined awareness levels to signify the level of cooperation.

We define cooperation using a matrix of required and available awareness levels for various human roles. This will then be correlated to user satisfaction levels. If there 
is a relation between awareness and satisfaction levels, it will be necessary to work out a new design with the required awareness levels. Since this is a new experimental model, it may be necessary to try a number of awareness related parameters, before one or a combination is found useful in this task domain.

\subsection{Awareness Levels}

The process of management is important for bringing out effective management solutions, and process knowledge consists of task knowledge and context knowledge (Duff, 1993). While task knowledge relates to the specification, design and performance of the task, context knowledge relates to rest of the process knowledge including relationships between various roles, tasks, and the environment of the task. In order to characterise these aspects in a group environment, one needs to be concerned with levels of:

- task knowledge for a particular interaction,

- knowledge of people involved and their roles in the task within the organisation,

- knowledge of other preoccupations of these people within the department or division,

- knowledge of interrelationship of various tasks in the department/division,

- knowledge of other activities of people concerned with the task (external to the organisation).

We define these using a parameter called "awareness level". This is likely to be the most important parameter to characterise awareness in an interaction. According to this definition, levels of cooperation (in increasing order) in a particular work context can be expressed in terms of several levels of awareness as follows:

- Level 0: concerns knowledge related to the given interaction.

- Level 1: level $0+$ Knowledge related to contact address of people involved in interaction.

- Level 2: Level $1+$ Knowledge related to contact address of all people in the interaction context.

- Level 3: Level 1+ Knowledge of activities of people involved in the interaction.

- Level 4: Level 2+ Knowledge of activities of all people involved in the interaction context.

In a group situation, required awareness for different members of a group can be different. Since the requirement for this information varies from task to task, and interaction to interaction, people need to keep switching awareness levels. As stated in the definition of Soft Systems Methodology, one needs to go through a number of iterations to arrive at a well-formed abstract root definitions (Avison, 1995).

Figure 3 shows the process of arriving at a CSCW design based on the cooperation analysis of real-life scenarios. We explore modelling interactions using: 
- Required and Supported Awareness levels of various roles in an interaction,

- Support for transition of awareness, and

- Tools to support the required awareness levels.

We expressed the problems in different scenarios in terms of the above model i.e. in terms of what is the required level of awareness, what is supported by existing tools. This results in a matrix of awareness levels for various interactions. Table 1 shows only interactions with low satisfaction level (less than 5) for the sample scenario (Figure 2). Details are available in (Ray, 1996).

Table 1: Awareness Matrix for the Scenario

\begin{tabular}{llll}
\hline Interaction & $\begin{array}{l}\text { Awareness } \\
\text { Required }\end{array}$ & $\begin{array}{l}\text { Awareness } \\
\text { Supported }\end{array}$ & $\begin{array}{l}\text { User Satisfaction } \\
\text { Level }\end{array}$ \\
\hline 2 & $3-2$ & $1-2$ & 3 \\
3 & $4-1$ & $3-0$ & 3 \\
4 & $3-3$ & $1-0$ & 3 \\
5 & $3-4$ & $1-3$ & 3 \\
6 & $4-3$ & $3-1$ & 2 \\
\hline
\end{tabular}

The next step is to develop schemes to realize required levels of awareness using group interaction databases, communication mechanisms and autonomous agents (system design part of CoMEN). How do we know if this would really provide better cooperation? There should be a correlation between satisfaction levels and awareness levels for different interactions. (Ray, 1997) analyses the interactions (in the scenario described earlier) for which the satisfaction is low (less than 5).

\subsection{Cooperative System Design Framework}

We have used the required awareness levels to derive a generic framework for cooperative system design (Figure 4). This was implemented using Lotus NOTES based groupware and a distributed object-oriented network management platform, called Cabletron SPECTRUM. SPECTRUM provides a distributed object model and some domain specific middleware. Details are available in (Ray, 1996). The system was subjected to heuristic evaluation based on contextual interview. This involved interviewing a number of experts in the application domain, using the prototype implementation as the context. We had to develop some broad evaluation criteria related to the application domain for this purpose. That led to the development of a questionnaire that was used to guide the interview. Details are available in (Ray, 1996).

The results of this study indicate that the awareness model is quite promising for the development of cooperative management solutions for enterprise networks. 
However, more research is needed to establish the findings in other application domains.

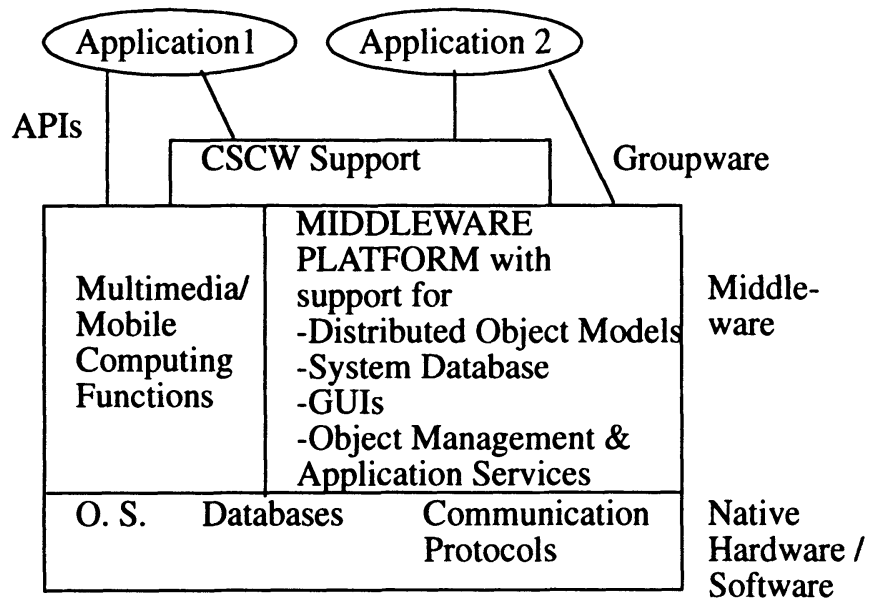

Figure 4 A Framework for Cooperative System Design

\subsection{Awareness Based Specification}

The results of this study suggest that lower user satisfaction level could be caused by an inability to support the right level of awareness. It may be noted that the definition of awareness level is imprecise due to the evolving nature of this theory. Also this allows the adjustment of definitions iteratively for a particular problem domain.

Given this outcome, we now undertake a high-level design of a cooperative management system for enterprise networks on the basis of required awareness levels. The idea is to come up with a conceptual design followed by a prototype implementation for evaluation. However, this presents a number of interesting problems:

1. The implementation of the desired awareness level may be in conflict with security requirements. This suggests that further studies could be conducted on issues, such as organisational security vs. cooperation, highest awareness level permitted by security considerations, and dynamic allocation of awareness level from interaction to interaction.

2. Awareness may not be properly usable. For example, large amounts of information may not increase awareness. Human users need the right tools at the right place and time (space-time matrix). This points to some communication requirements, such as multimedia visualisation, mobile computer based notification agents, etc. 
3. Difficulties in the implementation of required changes in levels of awareness.

4. Awareness of human vs. agent representing human role.

5. Effectiveness of available tools for awareness control, such as interaction level access controls, dynamic interaction databases, and autonomous agents.

Solutions to many of the problems are complex and may need more in-depth study.

\section{CONCLUSION}

In this paper, we have presented our new Cooperative systems development Methodology for Enterprise Networks (CoMEN). This methodology is based on Checkland's Soft Systems Methodology, and it adds mechanisms for modelling dynamic behaviour in a CSCW environment. This has lead to the definition of a new model for support for cooperation based on a measure called awareness levels.

This methodology was illustrated by a case study in a large telecommunication organisation to validate this methodology. This involved the study of representative problem scenarios in consultation with people working in the organisation under study. Each scenario was modelled using human roles, their interactions, and collaborative support tools. We presented for illustration one such scenario in this paper. The results indicated that low user satisfaction levels are caused by inadequate support for awareness. This provided an initial check for CoMEN. However, more study is required to prove its effectiveness in various types of cooperative business processes.

Although our study has been undertaken on cooperative management of networks, we feel some of the results are applicable to many other service industries that use help-desk based systems for customer support. Therefore, CoMEN has laid the foundation for further research on the development of effective methodologies for the development and deployment of distributed cooperative systems.

\section{ACKNOWLEDGEMENTS}

The author would like to thank Prof. Igor Hawryszkiewycz, Associate Professor Michael Fry, and Mr. Farhad Daneshgar of the University of Technology, Sydney, for valuable inputs in the development of this methodology.

\section{REFERENCES}

Avison, D. and Fitzgerald, G. (1995). Information Systems Development: Methodologies, Techniques and Tools. McGraw-Hill, 2nd Edition.

Benford, S. et al. (1994). Supporting Cooperative Work in Virtual Environments, The Computer Journal, $37,8$.

Checkland, P. et al. (1990). Soft System Methodologies in Action. Wiley, Chichester. CSCW (1994). Computer Supported Cooperative Work, Issue No 1, Published 
(electronically) by DTI in association with Noiseworks.

Dourish, P. et al. (1992). Awareness and Coordination in Shared Workspace, Proceedings of CSCW'92.

Duff, J. et al. (1993). Process Management: The Future of Integrated Management Systems, Proceedings of the Third International Symposium on Integrated Network Management (ISINM III).

Georgakopoulos, D. et al. (1995). An Overview of Workflow Management: From Process Modelling to Workflow Automation Infrastructure, Distributed and Parallel Databases, 3, 119-153, Kluwer Academic Publishers, Netherlands.

Grudin, J. (1994). Computer Supported Cooperative Work: History and Focus, IEEE Computer, May 1994.

Johnson, D. (1992). NOC Internal Integrated Trouble Ticket System Functional Specification Wishlist, Internet RFC 1297.

Lotus Development Corporation (1993.) Application Developer's Reference. Lotus Notes Release 3.

Preece, J. et al. (1994). Human - Computer Interaction. Addison-Wesley.

Ray, P. (1996). Cooperation Management of Enterprise Networks, PhD thesis, School of Computing Sciences, University of Technology, Sydney, Australia.

Ray, P. (1997). Integrated Management of Enterprise Networks: Group Cooperation

Perspective, IEICE Transactions on Communications, E80-B, 6.

Rumbaugh, J. et al. (1991). Object-Oriented Modelling and Design. Prentice Hall.

Sachs, P. (1995). Transforming Work: Collaboration, Learning, and Design, Communications of the ACM, 38, 9 .

Suchman, L.A. (1991). Plans and Situated Actions: The Problem of human machine communication. Cambridge University Press.

Winograd, T. et al. (1987). Understanding Computers and Cognition: A New Foundation for Design. Addison-Wesley.

\section{BIOGRAPHY}

Pradeep Ray is a member of the Academic Staff in Computing at the University of Western Sydney, Nepean, Australia. He has a MSc in Electrical Eng. from the Indian Institute of Technology, Kanpur, India, and a $\mathrm{PhD}$ in Computing Sciences from the University of Technology, Sydney. Prior to joining the University of Western Sydney, Nepean, Pradeep was a Senior Research Scientist with the CRC for Distributed Systems Technology (DSTC), Australia. He has had more than ten years experience in the international telecommunication industry on the development of enterprise networks. His research interests include network/ systems/services management, mobile computing, distributed/cooperative systems, and networked enterprise services, such as telemedicine. Pradeep organised the 8th IFIP/IEEE Intl. Workshop on Distributed Systems Operations and Management (DSOM'97) in October '97 as the Program Chair. He is a member of IEEE and ACM. 